\title{
The differential-symbol method of constructing the quasi-polynomial solutions of two-point problem
}

https://doi.org/10.1515/dema-2019-0010

Received October 13, 2018; accepted January 25, 2019

Abstract: The solvability of the problem with local nonhomogeneous two-point in time conditions for a homogeneous PDE of the second order in time and infinite order in spatial variable in the case when the set of zeroes of the characteristic determinant is not empty and does not coincide with $\mathbb{C}$ is investigated. The existence of a solution of the problem in which the right-hand sides of the two-point conditions are quasi-polynomials is proved. We propose the differential-symbol method of constructing the solutions of the problem.

Keywords: two-point conditions, characteristic determinant of a problem, differential-symbol method, quasipolynomial solutions

MSC: 35G15

\section{Introduction}

Many physical, economic, demographic and medical-biological processes can be described by models with multipoint in time conditions for PDEs and systems of them. The problems with such conditions have a simple interpretation of process observations at different points in time. For example, the two-point problem

$$
\begin{aligned}
& \frac{\partial^{2} U}{\partial t^{2}}-a^{2} \frac{\partial^{2} U}{\partial x^{2}}=0, \quad t \in \mathbb{R}, \quad x \in \mathbb{R}, \quad a \in \mathbb{R} \backslash\{0\}, \\
& U(0, x)=\varphi_{1}(x), U(h, x)=\varphi_{2}(x), \quad x \in \mathbb{R},
\end{aligned}
$$

describes the process of oscillation of an infinite string when the profile of the string is given at two moments of time $t=0$ and $t=h>0$. Unlike the Cauchy problem for equation (1) with zero initial conditions

$$
U(0, x)=\frac{\partial U}{\partial t}(0, x)=0,
$$

which has only a trivial solution, the two-point problem for equation (1) with zero two-point conditions

$$
U(0, x)=U(h, x)=0
$$

has nontrivial solutions of the form

$$
U(t, x)=\sin \frac{\pi t}{h} \cos \frac{\pi x}{a h} .
$$

Let us notice that the initial conditions, namely the conditions in which $U(0, x)$ and $\frac{\partial U}{\partial t}(0, x)$ are given at the one moment of time, are idealized. If a profile of a string for a oscillatory process is given at one moment

\footnotetext{
Zinovii Nytrebych: Lviv Polytechnic National University, Lviv, Ukraine; E-mail: znytrebych@gmail.com ^Corresponding Author: Oksana Malanchuk: Danylo Halytsky Lviv National Medical University, Lviv, Ukraine; E-mail: Oksana.Malan@gmail.com
} 
of time and the speed of change of the profile is given at another moment of time (although very close), then we obtain the problem for equation (1) with two-point conditions

$$
U(0, x)=\varphi_{1}(x), \frac{\partial U}{\partial t}(h, x)=\varphi_{2}(x)
$$

or

$$
U(h, x)=\varphi_{1}(x), \frac{\partial U}{\partial t}(0, x)=\varphi_{2}(x) .
$$

Obviously, the two-point in time conditions (5) and (6) are a partial case of the following conditions

$$
\begin{aligned}
& A_{1} U(0, x)+A_{2} \frac{\partial U}{\partial t}(0, x)=\varphi_{1}(x), \\
& B_{1} U(h, x)+B_{2} \frac{\partial U}{\partial t}(h, x)=\varphi_{2}(x),
\end{aligned}
$$

where $A_{1}, A_{2}, B_{1}, B_{2} \in \mathbb{C}$. Conditions (5) are obtained from (7) for $A_{1}=1, A_{2}=0, B_{1}=0, B_{2}=1$ and conditions (6) if $A_{1}=0, A_{2}=1, B_{1}=1, B_{2}=0$.

Therefore, the study of the solvability of the problem for a PDE with multipoint in time conditions is important for constructing a general theory of boundary-value problems and for practical problems.

Let us note the problems with multipoint in time conditions for PDEs are a generalization of problems with $n$-point conditions for ODEs which are known in the literature as Valle-Poussin problems [1-3]. The formulation and the first important results, which regard the solving of $n$-point problems for PDEs, are obtained in [4]. More general results of the solvability of multipoint problems in bounded domains for PDEs and systems of them are obtained in many works (see, for example, [5-8] and the bibliographies in them). Papers [9-11] are devoted to the establishment of the classes of uniqueness of the solution of the problems with multipoint in time conditions in unbounded domains.

In recent years the multipoint in time conditions become more general and get a new content. In particular, in conditions (7) instead of constants $A_{1}, A_{2}, B_{1}$, and $B_{2}$ arbitrary differential polynomials of spatial variables can be considered. Similar conditions are present in works [12,13].

Many works of scientists [14-16] are devoted to constructing the polynomial and quasi-polynomial solutions for the equations and boundary-value problems for them. Let us note that function (4), which is a nontrivial solution to the homogeneous problem (1), (3) is quasi-polynomial, which can be written in the form

$$
U(t, x)=A e^{\alpha t}\left(e^{\beta x}+e^{-\beta x}\right)-A e^{-\alpha t}\left(e^{\beta x}+e^{-\beta x}\right),
$$

where $A=-0,25 i, \alpha=i \pi h^{-1}, \beta=i \pi a^{-1} h^{-1}, i^{2}=-1$.

In unbounded domain of variables $t \in\left(T_{0}, T_{1}\right), x \in \mathbb{R}^{s}(s \in \mathbb{N})$, the differential-symbol method turned out to be convenient for solving the problem of the form

$$
\begin{aligned}
& \frac{\partial^{n} U}{\partial t^{n}}+\sum_{j=1}^{n} A_{j}\left(\frac{\partial}{\partial x}\right) \frac{\partial^{n-j} U}{\partial t^{n-j}}=f(t, x), \\
& l_{k} U(t, x)=\varphi_{k}(x), \quad k=\overline{1, n},
\end{aligned}
$$

in which $A_{1}\left(\frac{\partial}{\partial x}\right), \ldots, A_{n}\left(\frac{\partial}{\partial x}\right)$ are differential polynomials in spatial variables, $l_{1}, \ldots, l_{n}$ are linear functionals in the variable $t,-\infty \leq T_{0}<T_{1} \leq \infty$. The ideas behind this method are described in [17]. This differentialsymbol method allows establishing the classes of entire functions as classes of existence and uniqueness of the solution of problem (8), (9) in the form of actions of the differential expressions, whose symbols are the right-hand sides of conditions (9) and the right-hand side of equation (8), onto some functions of the parameter $\lambda$ and the vector-parameter $v$ and after the actions the parameters are set to zero. It means the solution of the problem can be found according to this method in the following form

$$
U(t, x)=\left.f\left(\frac{\partial}{\partial \lambda}, \frac{\partial}{\partial v}\right)\{G(t, x, \lambda, v)\}\right|_{\lambda=0, v=0}+\left.\sum_{k=1}^{n} \varphi_{k}\left(\frac{\partial}{\partial v}\right)\left\{T_{k}(t, x, v)\right\}\right|_{\nu=0},
$$


where $G(t, x, \lambda, v), T_{1}(t, x, v), \ldots, T_{n}(t, x, v)$ are functions which are especially constructed for each specific problem, $O=(0, \ldots, 0)$. The problems for PDEs with conditions in time variables by using the differentialsymbol method were studied in [18-21].

Symbol calculus which is inherent for differential-symbol method we can see, for example, in papers [22, 23]. In particular, for finding the definite integral $\int_{a}^{b} f(x) d x$, the indefinite integral $\int_{-\infty}^{0} f(x) d x$, and for Laplace's transform $L[f(x)]=\int_{0}^{+\infty} e^{-x y} f(y) d y$ authors propose formulas:

$$
\begin{aligned}
& \int_{a}^{b} f(x) d x=\lim _{\varepsilon \rightarrow 0} f\left(\frac{\partial}{\partial \varepsilon}\right) \frac{e^{\varepsilon b}-e^{\varepsilon a}}{\varepsilon}, \\
& \int_{-\infty}^{0} f(x) d x=\lim _{\varepsilon \rightarrow 0} f\left(\frac{\partial}{\partial \varepsilon}\right) \frac{1}{\varepsilon}, \\
& L[f(x)]=f\left(-\frac{\partial}{\partial x}\right) \frac{1}{x} .
\end{aligned}
$$

We note that in ([17], p. 26) the formula (10) is presented in the following form

$$
\int_{\beta_{1}}^{\beta_{2}} f(x) d x=\left.f\left(\frac{\partial}{\partial v}\right) \frac{e^{v \beta_{2}}-e^{v \beta_{1}}}{v}\right|_{v=0} .
$$

The present work continues the research of [24-27] and is devoted to the generalized problem (1), (7). The question of existence of solutions of the problem for the equation of the second order in time and generally infinite order in spatial variable with two-point conditions (7) in which $A_{1}, A_{2}, B_{1}, B_{2}$ are operator coefficients in the variable $x$ is being studied.

\section{Problem statement}

In the domain $(t, x) \in \mathbb{R}^{2}$, we investigate the solvability of the following two-point problem

$$
\begin{gathered}
L\left(\frac{\partial}{\partial t}, \frac{\partial}{\partial x}\right) U(t, x) \equiv \frac{\partial^{2} U}{\partial t^{2}}+a_{1}\left(\frac{\partial}{\partial x}\right) \frac{\partial U}{\partial t}+a_{2}\left(\frac{\partial}{\partial x}\right) U=0, \\
l_{k} U(t, x) \equiv b_{k 1}\left(\frac{\partial}{\partial x}\right) U(k h, x)+b_{k 2}\left(\frac{\partial}{\partial x}\right) \frac{\partial U}{\partial t}(k h, x)=\varphi_{k}(x), k \in\{0,1\},
\end{gathered}
$$

where $a_{1}\left(\frac{\partial}{\partial x}\right), a_{2}\left(\frac{\partial}{\partial x}\right)$ are the differential expressions of finite or infinite orders, symbols of which are the entire functions $a_{1}(v), a_{2}(v)$ for $v \in \mathbb{C}, b_{01}\left(\frac{\partial}{\partial x}\right), b_{02}\left(\frac{\partial}{\partial x}\right), b_{11}\left(\frac{\partial}{\partial x}\right)$, and $b_{12}\left(\frac{\partial}{\partial x}\right)$ are differential polynomials with complex coefficients. Moreover, their symbols $b_{01}(v), b_{02}(v), b_{11}(v)$ and $b_{12}(v)$, for each $v \in \mathbb{C}$ and $k \in\{0,1\}$, satisfy the conditions $\left|b_{k 1}(v)\right|^{2}+\left|b_{k 2}(v)\right|^{2} \neq 0$, where $\varphi_{0}(x), \varphi_{1}(x)$ are given entire functions (at least one of them is nonzero), $h$ is an arbitrary positive number.

The solution of problem (11), (12) is an entire function of the form

$$
U(t, x)=\sum_{\widetilde{k} \in \mathbb{Z}_{+}^{2}} u_{\widetilde{k}} t^{k_{0}} x^{k}, \quad \widetilde{k}=\left(k_{0}, k\right), \quad u_{\widetilde{k}} \in \mathbb{C},
$$

in variables $t$ and $x$ which satisfies equation (11) in domain $\mathbb{R}^{2}$ and conditions (12) in $\mathbb{R}$.

Let us write down corresponding ODE for the PDE (11)

$$
L\left(\frac{d}{d t}, v\right) T(t, v) \equiv \frac{d^{2} T}{d t^{2}}+a_{1}(v) \frac{d T}{d t}+a_{2}(v) T=0,
$$

where by $\left\{T_{0}(t, v), T_{1}(t, v)\right\}$ we denote the normal at the point $t=0$ fundamental system of solutions to the ODE (13). 
Let us consider the determinant of the form

$$
\Delta(v)=\left|\begin{array}{cc}
b_{01}(v) & b_{02}(v) \\
b_{11}(v) T_{0}(h, v)+b_{12}(v) \frac{d T_{0}}{d t}(h, v) & b_{11}(v) T_{1}(h, v)+b_{12}(v) \frac{d T_{1}}{d t}(h, v)
\end{array}\right| .
$$

The solvability of problem (11), (12) depends on the values of the determinant $\Delta(v)$. This determinant is called the characteristic determinant of problem (11), (12).

For a subset $M$ of the space $\mathbb{C}$ we consider the following classes of quasi-polynomials: $-K_{M}$ is the class of quasi-polynomials of the form

$$
\varphi(x)=\sum_{j=1}^{m} Q_{j}(x) e^{\alpha_{j} x}, \quad x \in \mathbb{R}, \quad m \in \mathbb{N},
$$

where $\alpha_{1}, \ldots, \alpha_{m}$ are pairwise distinct complex numbers in $M$ and $Q_{1}(x), \ldots, Q_{m}(x)$ are nonzero polynomials with complex coefficients;

$-K_{\mathbb{C}, M}$ is the class of quasi-polynomials of the form

$$
f(t, x)=\sum_{j=1}^{m} \sum_{l=1}^{N} P_{l j}(t, x) e^{\beta_{l} t+\alpha_{j} x}, \quad m, N \in \mathbb{N},
$$

where $P_{11}(t, x), \ldots, P_{N m}(t, x)$ are nonzero polynomials in variables $t, x$ with complex coefficients, $\beta_{1}, \ldots, \beta_{N}$ are pairwise distinct complex numbers and $\alpha_{1}, \ldots, \alpha_{m}$ are pairwise distinct complex numbers which belong to $M$.

Let us denote

$$
M=\{v \in \mathbb{C}: \Delta(v)=0\} .
$$

In this work we study the case $M \neq \varnothing$ and $M \neq \mathbb{C}$, and the right-hand sides $\varphi_{0}(x)$ and $\varphi_{1}(x)$ of the twopoint conditions (12) can belong to $K_{M}$. Note that the cases $M=\varnothing$ and $M=\mathbb{C}$ are investigated in [21, 24, 27]. Moreover, in [21] the existence of the unique solution of problem (11), (12) in the class $K_{\mathbb{C}, \mathbb{C} \backslash M}$ is proved when $\varphi_{0}(x)$ and $\varphi_{1}(x)$ belong to $K_{\mathbb{C} \backslash M}$ and the following formula is proposed for solving problem (11), (12):

$$
U(t, x)=\left.\sum_{k=0}^{1} \varphi_{k}\left(\frac{\partial}{\partial v}\right)\left\{\widetilde{T}_{k}(t, v) e^{v x}\right\}\right|_{v=0},
$$

where $\widetilde{T}_{0}(t, v), \widetilde{T}_{1}(t, v)$ are functions of the form

$$
\begin{aligned}
& \widetilde{T}_{0}(t, v)=e^{-\frac{1}{2} a_{1}(v)(t+h)} \frac{\left(b_{11}(v)-\frac{1}{2} a_{1}(v) b_{12}(v)\right) S_{h-t}(v)+b_{12}(v) C_{h-t}(v)}{\Delta(v)}, \\
& \widetilde{T}_{1}(t, v)=e^{-\frac{1}{2} a_{1}(v) t} \frac{\left(b_{01}(v)-\frac{1}{2} a_{1}(v) b_{02}(v)\right) S_{t}(v)-b_{02}(v) C_{t}(v)}{\Delta(v)},
\end{aligned}
$$

in which $S_{h-t}(v)=\frac{\sinh [(h-t) D(v)]}{D(v)}, C_{h-t}(v)=\cosh [(h-t) D(v)], D(v)=\frac{1}{2} \sqrt{a_{1}^{2}(v)-4 a_{2}(v)}$, $S_{t}(v)=\frac{\sinh [t D(v)]}{D(v)}, C_{t}(v)=\cosh [t D(v)]$.

It should be noted that, in formulas (16), the substituting $v=0$ and $v=\alpha_{j}$ is done after the action of the differential expressions by the parameter $v$.

The functions $\widetilde{T}_{0}(t, v), \widetilde{T}_{1}(t, v)$ are solutions of the ODE (13) and satisfy the conditions

$$
b_{k 1}(v) \widetilde{T}_{j}(k h, v)+b_{k 2}(v) \frac{d \widetilde{T}_{j}}{d t}(k h, v)=\delta_{k j}, \quad j, k \in\{0,1\},
$$

where $\delta_{k j}$ is the Kronecker delta. 
Also, we note that the action of the differential expression $\varphi\left(\frac{\partial}{\partial v}\right)$, symbol of which is the quasi-polynomial $\varphi(x)$ of the form (15), in formula (16) we understand as follows:

$$
\left.\varphi_{k}\left(\frac{\partial}{\partial v}\right)\left\{\widetilde{T}_{k}(t, v) e^{v x}\right\}\right|_{v=0}=\left.\sum_{j=1}^{m} Q_{j}\left(\frac{\partial}{\partial v}\right)\left\{\widetilde{T}_{k}(t, v) e^{v x}\right\}\right|_{v=\alpha_{j}} .
$$

Obviously, if $\varphi_{0}(x)$ and $\varphi_{1}(x)$ belong to $K_{\mathbb{C} \backslash M}$, then we do not get zero $\Delta(v)$.

This article is devoted to the case when at least one of the functions $\varphi_{0}(x)$ or $\varphi_{1}(x)$ belongs to $K_{M}$. We will prove that a solution of problem (11), (12) exists in the class $K_{\mathbb{C}, M}$ for $\varphi_{0}, \varphi_{1} \in K_{M}$. This solution is not unique in $K_{\mathbb{C}, M}$, since nontrivial solutions of equation (11) which satisfy the homogeneous conditions

$$
l_{k} U(t, x)=0, \quad k \in\{0,1\},
$$

exist in this class [24].

In the case $\varphi_{0}, \varphi_{1} \in K_{M}$, the question regarding the solvability of problem (11), (12) was not investigated. So, in this work we propose the differential-symbol method for constructing the solutions of problem (11), (12), if $\varphi_{0}(x)$ or $\varphi_{1}(x)$ belongs to $K_{M}$.

\section{Main results}

Let $\varphi_{0}, \varphi_{1} \in K_{M}$ and have the form

$$
\varphi_{k}(x)=Q_{k}(x) e^{\alpha_{k} x}, \quad k \in\{0,1\},
$$

where $\alpha_{0}, \alpha_{1} \in M$, that is $\alpha_{0}, \alpha_{1}$ are zeroes of $\Delta(v)$ of multiplicities $p_{\alpha_{0}}$ and $p_{\alpha_{1}}$ respectively $\left(p_{\alpha_{0}}, p_{\alpha_{1}} \in \mathbb{N}\right)$, $Q_{0}(x), Q_{1}(x)$ are polynomials of powers $n_{0}$ and $n_{1}$ respectively $\left(n_{0}, n_{1} \in \mathbb{Z}_{+}\right)$.

Theorem 3.1. Let the right-hand sides $\varphi_{0}(x)$ and $\varphi_{1}(x)$ of conditions (12) belong to $K_{M}$ and have the form (18). Then the solution of problem (11), (12) in the class of quasi-polynomials $K_{\mathbb{C}, M}$ exists and it can be written in the form

$$
U(t, x)=\left.\sum_{j=0}^{1} \frac{\left(\frac{\partial}{\partial v}\right)^{\left(n_{j}+1\right) p_{\alpha_{j}}} \rho_{j}(t, x, v)}{\left(\frac{d}{d v}\right)^{\left(n_{j}+1\right) p_{\alpha_{j}}} \Delta^{n_{j}+1}(v)}\right|_{v=\alpha_{j}},
$$

where

$$
\rho_{j}(t, x, v)=\Delta^{n_{j}+1}(v) Q_{j}\left(\frac{\partial}{\partial v}\right)\left\{\widetilde{T}_{j}(t, v) e^{v x}\right\}, \quad j \in\{0,1\} .
$$

Proof. It should be noted, that in the formula (20) the differential expression $Q_{j}\left(\frac{\partial}{\partial \nu}\right)$ is obtained from the polynomial $Q_{j}(x)$ by substituting $x$ with $\frac{\partial}{\partial v}$. First we note that the functions (20) are entire functions. Moreover, these functions are solutions to equation (11). Really, for $j \in\{0,1\}$ we have

$$
\begin{aligned}
L\left(\frac{\partial}{\partial t}, \frac{\partial}{\partial x}\right) \rho_{j}(t, x, v) & =\Delta^{n_{j}+1}(v) L\left(\frac{\partial}{\partial t}, \frac{\partial}{\partial x}\right) Q_{j}\left(\frac{\partial}{\partial v}\right)\left\{\widetilde{T}_{j}(t, v) e^{v x}\right\} \\
& =\Delta^{n_{j}+1}(v) Q_{j}\left(\frac{\partial}{\partial v}\right)\left\{e^{v x} L\left(\frac{d}{d t}, v\right) \widetilde{T}_{j}(t, v)\right\}=0 .
\end{aligned}
$$

In this chain of equalities we used the fact that the functions $\widetilde{T}_{0}(t, v), \widetilde{T}_{1}(t, v)$ are solutions to the ODE (13) and the following equality

$$
L\left(\frac{\partial}{\partial t}, \frac{\partial}{\partial x}\right)\left\{\widetilde{T}_{j}(t, v) e^{v x}\right\}=e^{v x} L\left(\frac{d}{d t}, v\right) \widetilde{T}_{j}(t, v) .
$$

Let us now prove that the function which is defined by equality (19) satisfies the two-point conditions (11). Really, for $k \in\{0,1\}$ we obtain 


$$
\begin{aligned}
l_{k \partial_{x}} U(t, x) & =\left.\sum_{j=0}^{1} \frac{\left(\frac{\partial}{\partial v}\right)^{\left(n_{j}+1\right) p_{\alpha_{j}}} l_{k \partial_{x}} \rho_{j}(t, x, v)}{\left(\frac{d}{d v}\right)^{\left(n_{j}+1\right) p_{\alpha_{j}}} \Delta^{n_{j}+1}(v)}\right|_{v=\alpha_{j}} \\
& =\left.\left.\sum_{j=0}^{1} \frac{\left(\frac{\partial}{\partial v}\right)^{\left(n_{j}+1\right) p_{\alpha_{j}}}\left(\Delta^{n_{j}+1}(v) Q_{j}\left(\frac{\partial}{\partial v}\right)\left\{\delta_{j k} e^{v x}\right\}\right)}{\left(\frac{d}{d v}\right)^{\left(n_{j}+1\right) p_{\alpha_{j}}} \Delta^{n_{j}+1}(v)}\right|_{v=\alpha_{j}}\right|_{v=\alpha_{k}}=\left.Q_{k}(x) \frac{\left(\frac{\partial}{\partial v}\right)^{\left(n_{k}+1\right) p_{\alpha_{k}}}\left(\Delta^{n_{k}+1}(v) e^{v x}\right)}{\left(\frac{d}{d v}\right)^{\left(n_{k}+1\right) p_{\alpha_{k}}} \Delta^{n_{k}+1}(v)}\right|_{v=\alpha_{k}} \\
& =\frac{\left(\frac{\partial}{\partial v}\right)^{\left(n_{k}+1\right) p_{\alpha_{k}}}\left(\Delta^{n_{k}+1}(v) Q_{k}(x) e^{v x}\right)}{\left(\frac{d}{d v}\right)^{\left(n_{k}+1\right) p_{\alpha_{k}}} \Delta^{n_{k}+1}(v)} \\
& =Q_{k}(x) e^{\alpha_{k}(x)}=\varphi_{k}(x) .
\end{aligned}
$$

In these equalities we use the fact that $\left.\left(\frac{d}{d v}\right)^{s} \Delta^{n_{j}+1}(v)\right|_{v=\alpha_{k}}=0$ for arbitrary $s \in\left\{0, \ldots,\left(n_{k}+1\right) p_{\alpha_{k}}-1\right\}$.

Remark 3.1. In Theorem 3.1, we consider the functions $\varphi_{0}(x)$ and $\varphi_{1}(x)$ as monomial polynomials of the form (15) for $m=1$. However, if these functions have the form (15) for $m>1$, then by Theorem 3.1 we find the solution to problem (11), (12) for each term of the polynomial and the sum of found solutions will also be a solution to problem (11), (12).

Example 3.1. In domain $(t, x) \in \mathbb{R}^{2}$, to find the solutions of the two-point problem

$$
\begin{aligned}
& {\left[\frac{\partial^{2}}{\partial t^{2}}-2 \frac{\partial^{3}}{\partial x^{2} \partial t}+\frac{\partial^{4}}{\partial x^{4}}\right] U(t, x)=0,} \\
& \frac{\partial U}{\partial x}(0, x)+\frac{\partial U}{\partial t}(0, x)=x, \quad \frac{\partial U}{\partial x}(1, x)+\frac{\partial U}{\partial t}(1, x)=2 e^{-x} .
\end{aligned}
$$

For problem (21), (22), we have $a_{1}(v)=-2 v^{2}, a_{2}(v)=v^{4}, b_{01}(v)=b_{11}(v)=v, b_{02}(v)=b_{12}(v)=1$.

The characteristic determinant of problem (21), (22) and the set $M$ have the form

$$
\Delta(v)=v^{2}(v+1)^{2} e^{v^{2}}, \quad M=\{0 ;-1\} .
$$

For the function $\varphi_{0}(x)=x \in K_{M}$ we can write down $Q_{0}(x)=x, \alpha_{0}=0, p_{\alpha_{0}}=2, n_{0}=1$. For the function $\varphi_{1}(x)=2 e^{-x} \in K_{M}$ we have $Q_{1}(x)=2, \alpha_{1}=-1, p_{\alpha_{1}}=2, n_{1}=0$.

For problem (21), (22) the functions $\widetilde{T}_{0}(t, v), \widetilde{T}_{1}(t, v)$ have the following form

$$
\begin{aligned}
& \widetilde{T}_{0}(t, v)=e^{v^{2}(t+1)} \frac{\left(v^{2}+v\right)(1-t)+1}{\Delta(v)}, \\
& \widetilde{T}_{1}(t, v)=e^{v^{2} t} \frac{\left(v^{2}+v\right) t-1}{\Delta(v)} .
\end{aligned}
$$

Then we can write the functions $\rho_{0}(t, x, v), \rho_{1}(t, x, v)$ down as

$$
\begin{aligned}
& \rho_{0}(t, x, v)=\Delta^{2}(v) \frac{\partial}{\partial v}\left\{\widetilde{T}_{0}(t, v) e^{v x}\right\}, \\
& \rho_{1}(t, x, v)=2 \Delta(v)\left\{\widetilde{T}_{1}(t, v) e^{v x}\right\} .
\end{aligned}
$$

By formula (19), the solution of problem (21), (22) can be found as

$$
\begin{aligned}
U(t, x) & =\left.\frac{\left(\frac{\partial}{\partial v}\right)^{4} \rho_{0}(t, x, v)}{\left(\frac{d}{d v}\right)^{4} \Delta^{2}(v)}\right|_{v=0}+\left.\frac{\left(\frac{\partial}{\partial v}\right)^{2} \rho_{1}(t, x, v)}{\left(\frac{d}{d v}\right)^{2} \Delta(v)}\right|_{v=-1} \\
& =\frac{1}{6} x^{3}+2 x t-x-3+t-t^{2}-\frac{1}{2} x^{2}-\frac{1}{2} x^{2} t-x(x-2 t) e^{t-x-1}
\end{aligned}
$$


This solution is not unique in the class $K_{\mathbb{C}, M}$, since in [24] it is proved that the two-point problem for equation (21) with homogeneous conditions

$$
\frac{\partial U}{\partial x}(0, x)+\frac{\partial U}{\partial t}(0, x)=0, \quad \frac{\partial U}{\partial x}(1, x)+\frac{\partial U}{\partial t}(1, x)=0
$$

has nontrivial solutions of the form

$$
U(t, x)=c_{1}+c_{2}(x-t)+c_{3} e^{t-x}+c_{4}(x-t) e^{t-x},
$$

where $c_{1}, c_{2}, c_{3}, c_{4}$ are arbitrary complex constants.

Remark 3.2. If $\varphi_{0} \in K_{\mathbb{C} \backslash M}$ or $\varphi_{0}(x)=0$ and $\varphi_{1} \in K_{M}$, moreover if $\varphi_{1}$ has the form (18) for $k=1$, then the solution of problem (11), (12) in the class $K_{\mathbb{C}, \mathbb{C}}$ exists and can be found by the formula

$$
U(t, x)=\left.\varphi_{0}\left(\frac{\partial}{\partial v}\right)\left\{\widetilde{T}_{0}(t, v) e^{v x}\right\}\right|_{v=0}+\left.\frac{\left(\frac{\partial}{\partial v}\right)^{\left(n_{1}+1\right) p_{\alpha_{1}}} \rho_{1}(t, x, v)}{\left(\frac{d}{d v}\right)^{\left(n_{1}+1\right) p_{\alpha_{1}}} \Delta^{n_{1}+1}(v)}\right|_{v=\alpha_{1}} .
$$

Similarly, if $\varphi_{0} \in K_{M}$, moreover if $\varphi_{0}$ has the form (18) for $k=0, \varphi_{1} \in K_{\mathbb{C} \backslash M}$ or $\varphi_{1}(x)=0$, then the solution to problem (11), (12) in the class $K_{\mathbb{C}, \mathbb{C}}$ exists and can be found by the formula

$$
U(t, x)=\left.\frac{\left(\frac{\partial}{\partial v}\right)^{\left(n_{0}+1\right) p_{\alpha_{0}}} \rho_{0}(t, x, v)}{\left(\frac{d}{d v}\right)^{\left(n_{0}+1\right) p_{\alpha_{0}}} \Delta^{n_{0}+1}(v)}\right|_{\nu=\alpha_{0}}+\left.\varphi_{1}\left(\frac{\partial}{\partial v}\right)\left\{\widetilde{T}_{1}(t, v) e^{v x}\right\}\right|_{\nu=0} .
$$

Example 3.2. Let us find the solutions of the two-point problem for the differential-functional equation

$$
\begin{gathered}
\frac{\partial^{2} U}{\partial t^{2}}(t, x)-\left(1+\frac{\partial}{\partial x}\right)^{2} U(t, x+2)=0, \quad(t, x) \in \mathbb{R}^{2}, \\
\left(1+\frac{\partial}{\partial x}\right) U(0, x)+\frac{\partial^{2} U}{\partial t \partial x}(0, x)=e^{-x}, \\
\left(1+\frac{\partial}{\partial x}\right) U(h, x)+\frac{\partial^{2} U}{\partial t \partial x}(h, x)=x, \quad x \in \mathbb{R} .
\end{gathered}
$$

For problem (25), (26), we have $a_{1}(v)=0, a_{2}(v)=-(1+v)^{2} e^{2 v}, b_{01}(v)=b_{11}(v)=1+v, b_{02}(v)=b_{12}(v)=v$.

Let us write down the characteristic determinant of problem (25), (26) and the set of its zeroes in the form

$$
\begin{aligned}
& \Delta(v)=(v+1)\left(1-v^{2} e^{2 v}\right) e^{-v} \sinh \left[(v+1) e^{v} h\right], \\
& M=\left\{v \in \mathbb{C}:\left(1-v^{2} e^{2 v}\right) \sinh \left[(v+1) e^{v} h\right]=0\right\} \cup\{-1\} .
\end{aligned}
$$

The function $\varphi_{0}(x)=e^{-x}$ belongs to $K_{M}$, moreover $\alpha_{0}=-1, p_{\alpha_{0}}=2, n_{0}=0$. The function $\varphi_{1}(x)=x$ belongs to $K_{\mathbb{C} \backslash M}$.

The solution to problem (25), (26) we can find by formula (24):

$$
U(t, x)=\left.\frac{\left(\frac{\partial}{\partial v}\right)^{2} \rho_{0}(t, x, v)}{\left(\frac{d}{d v}\right)^{2} \Delta(v)}\right|_{v=-1}+\left.\varphi_{1}\left(\frac{\partial}{\partial v}\right)\left\{\widetilde{T}_{1}(t, v) e^{v x}\right\}\right|_{v=0},
$$

where the functions $\widetilde{T}_{0}(t, v), \widetilde{T}_{1}(t, v), \rho_{0}(t, x, v)$ are following:

$$
\begin{aligned}
\widetilde{T}_{0}(t, v) & =\frac{e^{-v} \sinh \left[(h-t)(1+v) e^{v}\right]+v \cosh \left[(h-t)(1+v) e^{v}\right]}{\Delta(v)}, \\
\widetilde{T}_{1}(t, v) & =\frac{e^{-v} \sinh \left[t(1+v) e^{v}\right]-v \cosh \left[t(1+v) e^{v}\right]}{\Delta(v)}, \\
\rho_{0}(t, x, v)=\Delta(v)\left\{\widetilde{T}_{0}(t, v) e^{v x}\right\} & =\left(e^{-v} \sinh \left[(h-t)(1+v) e^{v}\right]+v \cosh \left[(h-t)(1+v) e^{v}\right]\right) e^{v x} .
\end{aligned}
$$


Therefore,

$$
\begin{aligned}
U(t, x)= & \left.\frac{\left(\frac{\partial}{\partial v}\right)^{2}\left\{\left(e^{-v} \sinh \left[(h-t)(1+v) e^{v}\right]+v \cosh \left[(h-t)(1+v) e^{v}\right]\right) e^{v x}\right\}}{\left(\frac{d}{d v}\right)^{2}\left((v+1)\left(1-v^{2} e^{2 v}\right) e^{-v} \sinh \left[(v+1) e^{v} h\right]\right)}\right|_{v=-1} \\
& +\left.\frac{\partial}{\partial v} \frac{\left(e^{-v} \sinh \left[t(1+v) e^{v}\right]-v \cosh \left[t(1+v) e^{v}\right]\right) e^{v x}}{(v+1)\left(1-v^{2} e^{2 v}\right) e^{-v} \sinh \left[(v+1) e^{v} h\right]}\right|_{v=0} \\
= & \frac{-x^{2}+2 x(h-t)+2 x-e^{-2}(h-t)^{2}}{2 h\left(1-e^{-2}\right)} e^{-x} \\
& +\frac{1}{\sinh h}\left[(x-1) \sinh t+(2 t-1) \cosh t-2 h \frac{\sinh t}{\tanh h}\right]
\end{aligned}
$$

Let us note that the found solution of problem (25), (26) is not unique in the class $K_{\mathbb{C}, \mathbb{C}}$, since, for example, the functions

$$
U_{1}(t, x)=c_{1} e^{-x}, \quad U_{2}(t, x)=c_{2}(1-t-x) e^{-x}, \quad c_{1}, c_{2} \in \mathbb{C},
$$

are solutions to the corresponding homogeneous problem and the sum of each of these solutions with the found solution $U(t, x)$ is also a solution to problem (25), (26).

\section{Conclusions}

The solvability of the problem for the homogeneous partial differential equation of second order in time and finite or infinite order in spatial variable (11) with nonhomogeneous two-point in time conditions (12) in the case when the set of zeroes of the characteristic determinant of problem (11), (12) is not empty and does not coincide with $\mathbb{C}$ is investigated. If the right-hand sides of the two-point conditions are quasi-polynomials then the existence of solutions of the problem is proved and the formulas (19), (23), (24) for constructing these solutions are found. We show the application of the proposed differential-symbol method for some two-point problems (examples 3.1 and 3.2).

\section{References}

[1] Vallee-Poussin Ch. J., Sur l'equation differentielle lineaire du second ordre. Determination d'une integrale par deux valeurs assignees Extension aux equations d'ordre $n$, J. Math. Pures Appl. (9), 1929, 8, 125-144

[2] Picone M., Sui valori eccezionali di un parametro do cui dipend un equazione differentiale lineare ordinaria del secondo ordine, Pisa, 1909

[3] Tamarkin Ya. D., About the some general problem of the theory of ordinary differential equations and expansion of arbitrary functions in series, Pg., 1917

[4] Ptashnyk B. Yo., Problem of Vallee-Poussin type for hyperbolic equations with constant coefficients, DAN URSR, 1966, 10, 1254-1257

[5] Kiguradze T., The Vallee-Poussin problem for higher order nonlinear hyperbolic equations, Comput. Math. Appl., 2010, 59(2), 994-1002

[6] Ptashnyk B. Yo., Ill-posed boundary value problems for partial differential equations, Nauk. Dumka, Kyiv, 1984

[7] Ptashnyk B. Yo., Il'kiv V. S., Kmit I. Ya., Polishchuk V. M., Nonlocal boundary value problems for partial differential equations, Nauk. dumka, Kyiv, 2002

[8] Ptashnyk B. Yo., Symotyuk M. M., Multipoint problem for nonisotropic partial differential equations with constant coefficients, Ukr. Math. Journ., 2003, 55(2), 293-310

[9] Borok V. M., Uniqueness classes for the solution of a boundary problem in an infinite layer, Dokl. Akad. Nauk SSSR, 1968, 183(5), 995-998

[10] Borok V. M., Perelman M. A., Unique solution classes for a multipoint boundary value problem in an infinite layer, Izv. Vyss. Ucebn. Zaved. Matematika, 1973, 8, 29-34

[11] Vilents' I. L., The classes of uniqueness of the solution of a general boundary-value problem in a layer for systems of linear partial differential equations, Dop. Akad. Nauk URSR, 1974, 3, 195-197 
[12] Fardigola L. V., Well-posed problems in a layer with differential operators in boundary conditions, Ukr. Math. J., 1992, 44(8), 983-989

[13] Fardigola L. V., Nonlocal two-point boundary-value problems in a layer with differential operators in the boundary condition, Ukr. Math. J., 1995, 47(8), 1283-1289

[14] Hayman W. K., Shanidze Z. G., Polynomial solutions of partial differential equations, Methods Appl. Anal., 1999, 6(1), 97-108

[15] Hile G. N., Stanoyevitch A., Heat polynomial analogous for equations with higher order time derivatives, J. Math. Anal. Appl., 2004, 295, 595-610

[16] Pedersen P., A basis for polynomial solutions for systems of linear constant coefficient PDE's, Adv. Math., 1996, 117, 157-163

[17] Kalenyuk P., Nytrebych Z., Generalized scheme of separation of variables, Differential-symbol method, Publishing House of Lviv Polytechnic National University, Lviv, 2002

[18] Kalenyuk P. I., Kohut I. V., Nytrebych Z. M., Problem with integral condition for partial differential equation of the first order with respect to time, J. Math. Sci., 2012, 181(3), 293-304

[19] Nitrebich Z. M., A boundary-value problem in an unbounded strip, J. Math. Sci., 1996, 79(6), 1388-1392

[20] Nitrebich Z. M., An operator method of solving the Cauchy problem for a homogeneous system of partial differential equations, J. Math. Sci., 1996, 81(6), 3034-3038

[21] Nytrebych Z., Malanchuk 0., The differential-symbol method of solving the two-point problem with respect to time for a partial differential equation, J. Math. Sci., 2017, 224(4), 541-554

[22] Kempf A., Jackson D. M., Morales A. H., New Dirac delta function based methods with applications to perturbative expansions in quantum, J. Phys. Math. Theor., 2014, 47(41)

[23] Kempf A., Jackson D. M., Morales A. H., How to (parth) integrate by differentiating, J. Phys.: Conf. Ser., 2015, 626

[24] Malanchuk O., Nytrebych Z., Homogeneous two-point problem for PDE of the second order in time variable and infinite order in spatial variables, Open Math., 2017, 15, 101-110

[25] Nytrebych Z., Malanchuk O., Il'kiv V., Pukach P., Homogeneous problem with two-point in time conditions for some equations of mathematical physics, Azerb. J. Math., 2017, 7(2), 180-196

[26] Nytrebych Z., Il'kiv V., Pukach P., Malanchuk O., On nontrivial solutions of homogeneous Dirichlet problem for partial differential equations in a layer, Kragujevac J. Math., 2018, 42(2), 193-207

[27] Nytrebych Z., Malanchuk O., Il'kiv V., Pukach P., On the solvability of two-point in time problem for PDE, Italian J. Pure Appl. Math., 2017, 38, 715-726 